\title{
A PECULIAR VARIABLE STAR HD229221 - FROM 1994 TO 1997
}

\author{
JUN-JIE WANG AND JING-YAO HU \\ Beijing Astronomical Observatory, Chinese Academy of Sciences \\ wangjj@bao01.bao.ac.cn,hjy@bao01.bao.ac.cn
}

From 1994 to 1997, we observed the star HD229221 several times spectroscopically by using the Beijing Astronomical Observatory $2.16 \mathrm{~m}$ telescope and the spectrograph Universal.

From the spectra we clearly see that the H- $\alpha$ spectral feature of this star underwent a peculiar variation, from a single emission line to one composed of three components, i.e. emission+absorption+filled emission. In our paper, based on an analysis of its spectral line variation and some physical parameters (e.g. luminosity, age, etc.), we discuss the possible reasons that might cause this peculiar variation. 